University of Nebraska - Lincoln

DigitalCommons@University of Nebraska - Lincoln

2006

Stochastic Algorithm for Improved Operations at Isolated High Speed Intersections

\author{
Anuj Sharma \\ University of Nebraska - Lincoln, anujs@iastate.edu \\ Darcy M. Bullock \\ Purdue University, darcy@purdue.edu \\ Srinivas Peeta \\ Purdue University, peeta@purdue.edu
}

Follow this and additional works at: https://digitalcommons.unl.edu/civilengfacpub

Part of the Civil Engineering Commons

Sharma, Anuj; Bullock, Darcy M.; and Peeta, Srinivas, "Stochastic Algorithm for Improved Operations at Isolated High Speed Intersections" (2006). Civil Engineering Faculty Publications. 33.

https://digitalcommons.unl.edu/civilengfacpub/33

This Article is brought to you for free and open access by the Civil Engineering at DigitalCommons@University of Nebraska - Lincoln. It has been accepted for inclusion in Civil Engineering Faculty Publications by an authorized administrator of DigitalCommons@University of Nebraska - Lincoln. 


\title{
STOCHASTIC ALGORITHM FOR IMPROVED OPERATIONS AT ISOLATED HIGH SPEED INTERSECTIONS
}

\author{
Anuj Sharma ${ }^{1 a}$, Darcy Bullock ${ }^{\mathrm{b}}$, Srinivas Peeta ${ }^{\mathrm{c}}$, \\ ${ }^{a}$ Graduate Research Assistant; ${ }^{b}$ Professor; ${ }^{c}$ Associate Professor \\ School of Civil Engineering, Purdue University
}

\begin{abstract}
The current practice of specifying simultaneous gap out logic at isolated high speed signalized intersections places constraints on the signal controller logic that cannot be satisfied under high congestion level. Further, it often results in degraded signal efficiency and dilemma zone protection. A stochastic approach is proposed in this paper with the objective of increasing safety and efficiency at these intersections. Copyright $($ C 2006 IFAC
\end{abstract}

Keywords: Safety, Traffic Control, Detectors, Efficiency Enhancement, Road Traffic.

\section{INTRODUCTION}

Intersection crashes constitute a significant portion of total fatalities in the United States; they account for an average of 9,000 fatalities and 1.5 million injuries annually. Red light running (RLR) is a major cause of fatal and injury-related crashes. Also, motorists are more likely to be injured in such crashes. The National Highway Traffic Safety Administration of USA reported that in 2002 there were 921 fatalities and 178,000 injuries resulting from 207,000 crashes attributable to motorists running red lights at signalized intersections. A survey conducted by the U.S. Department of Transportation and the American Trauma Society indicates that 63 percent of Americans witness a RLR incident more than once a week and one in three Americans knows someone who has been injured or killed because of a red-light runner.

Rural high-speed isolated intersections are more susceptible to RLR crashes. Drivers travel at high speeds at such intersections with a high expectancy of proceeding through them without stopping. This expectancy is violated under dilemma zone incursions, leading to elevated risk of crashes. The most commonly implemented strategy to eliminate this problem is enabling simultaneous gap out logic.

Simultaneous gap out logic is adopted at isolated intersections to provide dilemma zone protection for

\footnotetext{
${ }^{1}$ Address: Department of Civil Engineering, Purdue University, West Lafayette, IN-47907.

Email: sharma23@purdue.edu.

Tel: 765-494-2206

Fax: 765-496-7996
}

the drivers on the primary street. It is widely believed that the simultaneous gap out logic provides $100 \%$ dilemma zone protection at an intersection. On the contrary, simultaneous gap out logic works well under low traffic volumes but the performance deteriorates under congested conditions. This paper will propose a stochastic approach to improve the performance of simultaneous gap out logic under medium to high traffic volumes.

\section{PROBLEM DESCRIPTION}

\subsection{Dilemma Zone}

The dilemma zone constitutes the area on the roadway where the driver is indecisive about whether to stop or to go on the onset of yellow interval (ITE, 1999). Figure 1 shows this concept graphically. Driver 1 in the "Can Go" zone can safely cross the intersection while staying within the speed limit. Driver 3 in "Can Stop" can come to a safe stop before the stop bar with a comfortable deceleration. Driver 2 in the "Dilemma Zone" can neither cross the intersection before the onset of red if he stays within speed limit nor can stop the vehicle by applying a comfortable deceleration. The concept of a dilemma zone appeared in studies by Gazis et al. (1960), Olson and Rothery (1972), Crawford (1962) and Herman (1963). Sheffi and Mahmassani (1981) identify the dilemma as the drivers' decision to proceed through the intersection or to stop when the signal indication changes from green to amber. Sheffi and Mahmassani (1981) further defined it as the zone within which the driver could neither come to a stop nor proceed through the intersection before the 


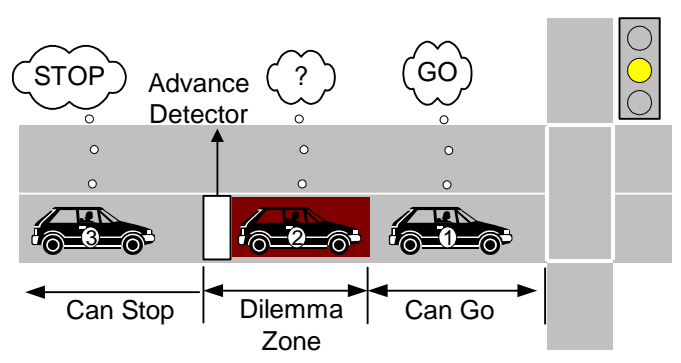

Fig. 1. The Dilemma zone

end of the amber phase. Zegeer (1977) proposed a probabilistic approach by defining a dilemma zone as the road segment where more than $10 \%$ and less than $90 \%$ of the drivers would choose to stop. Sheffi and Mahmassani (1981) developed dilemma zone curves of 'percent drivers stopping' versus 'distance from stop bar' at the instant when the signal indication changes from green to amber. Dilemma zone is also referred to as the "option zone" or the "zone of indecision” (McCoy and Pesti, 2002).

Occurrences of a dilemma zone incursion (presence of driver/drivers in the dilemma zone) elevate the risk of crashes. Dilemma zone incursions have also been identified as major causes of red light running and rear end collisions. Dilemma zone protection is provided to minimize, and if possible eliminate, the occurrences of dilemma zone incursions. This is usually accomplished by placing an advance vehicle detector just beyond the start of dilemma zone (as shown in Figure 1). Advance detector detects a vehicle and extends the green sufficiently to allow the vehicle to travel past the dilemma zone to the "Can Go" zone. Such an approach is often referred to as green extension system. A "before-and-after" evaluation (Zegeer and Deen, 1978) of the extension system on three intersections in Kentucky to determine their effect on crashes showed a 54 percent reduction in accidents per year at the three sites combined. The duration of the before-period was 8.5 years and the duration of the after-period was 3.7 years. There were 70 accidents in the beforeperiod and 14 accidents in the after-period.

The safety benefits of a green extension system are negated if the phase reaches their maximum green time and arbitrarily terminates (max out). The green extension system usually uses simultaneous gap out logic to pool the through lanes of high speed movement. This is done to ensure that none of the included lanes have vehicles in the dilemma zone under the normal termination of green phase. The simultaneous gap out logic works well during low volume conditions. However, the frequency of max out increases with the increase in traffic volume, jeopardizing both safety and efficiency of operations at the intersection.

Enhanced systems like the TTI truck priority system (Middleton, et al., 1997), intelligent detectioncontrol system (Bonneson, et al., 2002) etc. are the other forms of green extension/termination systems.
These systems, which promise improved dilemma zone protection but require expensive detection, are not widely used.

Other methodologies (Saito, et al., 1990) dynamically vary the clearance intervals (yellow clearance and all red) to minimize dilemma zone incursions. These methodologies have not been widely implemented or tested. They can be used as complementary to green extension systems. This paper focuses on the evaluation and improvement of simultaneous gap out logic which is the most commonly used feature (available in almost all the controllers) for dilemma zone protection. The concept of simultaneous gap out logic is explained hereafter.

\subsection{Simultaneous gap out logic}

As shown in Figure 2, in actuated control, phases 2 and 6 (main street through phases) are most often linked for gap out purposes. This imposes an additional constraint on the control system. The constraint requires that when crossing the barrier, phases 2 and 6 must gap out together in order to terminate the green interval. In the absence of simultaneous gap out logic, if phase 2 gaps out prior to phase 6 both the phases go to clearance as soon as

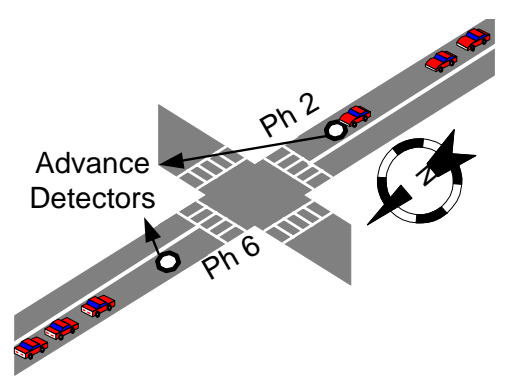

a) Example intersection

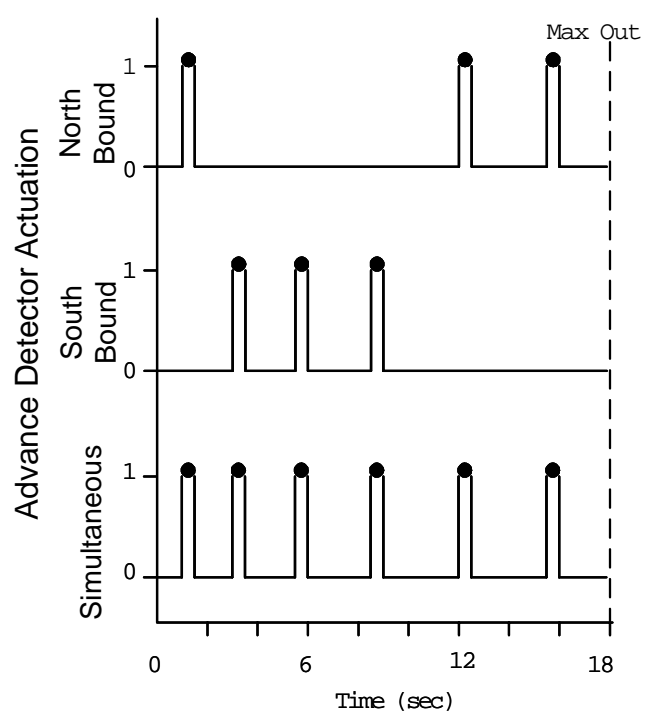

b) Example detector inputs

Fig. 2. Illustration of simultaneous gap out logic 
gap out logic, the advance detectors on these $n$ lanes are connected in series. This leads to the summation placed on phase 2. With simultaneous gap out enabled the new call will extend phase 2 even though it would have already gapped out. Here, phase 2 and phase 6 need to gap out simultaneously to end the phases. Hence, the simultaneous gap out logic inherently increases the likelihood of max out scenarios.

Figure 2 further illustrates the principle of simultaneous gap out logic for a hypothetical intersection. Figure 2a shows the snapshot of the hypothetical intersection with position of cars at time zero. Figure $2 \mathrm{~b}$ plots the time at which the advance detectors of north bound and south bound are actuated. The third plot from top in Figure 2b shows the actuations seen by the controller if the simultaneous gap out logic was implemented. An extension time of $4 \mathrm{sec}$ is assumed (with each actuation, green is extended by 4 seconds). The max out time is assumed to be 18 seconds. There are three vehicles in north bound direction passing the advance detector at time $1 \mathrm{sec}, 12 \mathrm{sec}$ and $16 \mathrm{sec}$ and three vehicles in south bound direction which are detected by the advance detector at time 3 sec, 5.5 sec and 9 sec. Suppose the north bound direction is serviced by phase 2 and phase 6 services south bound direction. If the simultaneous gap out logic is not implemented, phase 2 will gap out at 5 sec and phase 6 gaps out at $13 \mathrm{sec}$. Thus phases 2 and 6 enter the clearance interval at 13 seconds. However, as can be observed from the Figure 2, one vehicle at 12 seconds will be present in the dilemma zone. If instead, the simultaneous gap out logic were implemented, phases 2 and 6 keep extending until 18 seconds when the phase goes to the clearance interval due to max out. However, this also leads to one dilemma zone incursion. There would be no dilemma zone incursion if the max time were greater than 20 seconds. However, with a max out time setting of $18 \mathrm{sec}$ the simultaneous gap out logic drags the cycle length without providing any safety benefits.

The above example illustrates that simultaneous gap out logic can be problematic in cases of medium to high volumes. Under such scenarios it will reduce the efficiency of the intersection without any dilemma zone protection when the phases max out. The maxing out of phases leads to increase in cycle lengths. The increase in cycle length causes an increased delay on the intersection thereby increasing the travel time and vehicle operating costs.

\section{PROPOSED APPROACH}

Figure 3 compares the traditional approach and the proposed traffic adaptive approach for implementing gap out logic. In the traditional approach, all the lanes are included in the simultaneous gap out logic irrespective of the volume conditions in the field.

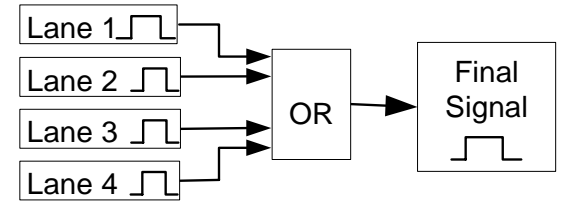

a) Traditional simultaneous gap out logic

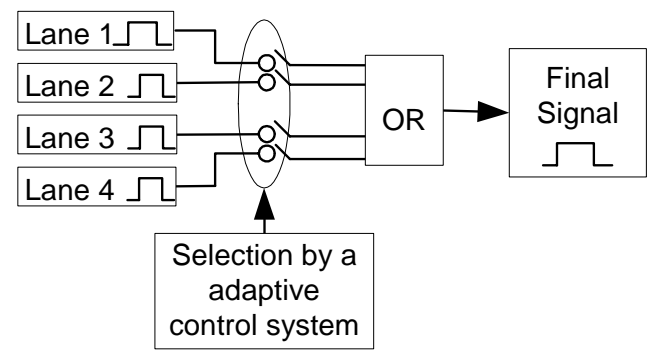

b) Proposed simultaneous gap out logic

Fig. 3. Traditional versus traffic adaptive approach for selecting number of lanes to be included in simultaneous gap out logic.

The OR block in Figure 3 implies that the detectors of all four lanes are connected in series and the resulting signal is evaluated for finding a gap out. The proposed approach will use traffic adaptive control logic to determine the number of lanes that should be included in the simultaneous gap out logic. A description of the proposed logic and its analysis is provided hereafter.

If the traffic arrivals are assumed to be Poisson distributed, then the inter arrival times between the vehicles will be negative exponentially distributed. When $\mathrm{n}$ lanes are to be included in the simultaneous gap out logic, the advance detectors on these $n$ lanes are connected in series. This leads to the summation of $n$ distributions (random variables) of traffic arrivals on the grouped lanes. By the principle of convolution (Casella and Berger, 2002), the sum of $n$ independent Poisson random variables with means $\lambda_{1}, \lambda_{2}, \lambda_{3} \ldots \lambda_{n}$ is also a Poisson distribution with mean $\sum_{i=1}^{n} \lambda_{i}$. For example, if 4 lanes, having independent exponentially distributed headways are included in the simultaneous gap out logic, the headway distribution of the resulting combination will also be an exponential distribution having volume equal to the sum of volumes of those 4 lanes.

Simulation runs were performed in Matlab to estimate the proportion of cycles that will max out under a given volume condition and maximum green time. This simulation assumed an exponential distribution of headways. The volumes were varied from $100 \mathrm{vph}$ to $8000 \mathrm{vph}$. The upper limit is chosen as capacity of 4 lanes (capacity of single lane is usually near 2000 vphpln). Green max times ranging from 30 seconds to 80 seconds were analyzed. Figure 4 shows the resulting plot from the simulation. 


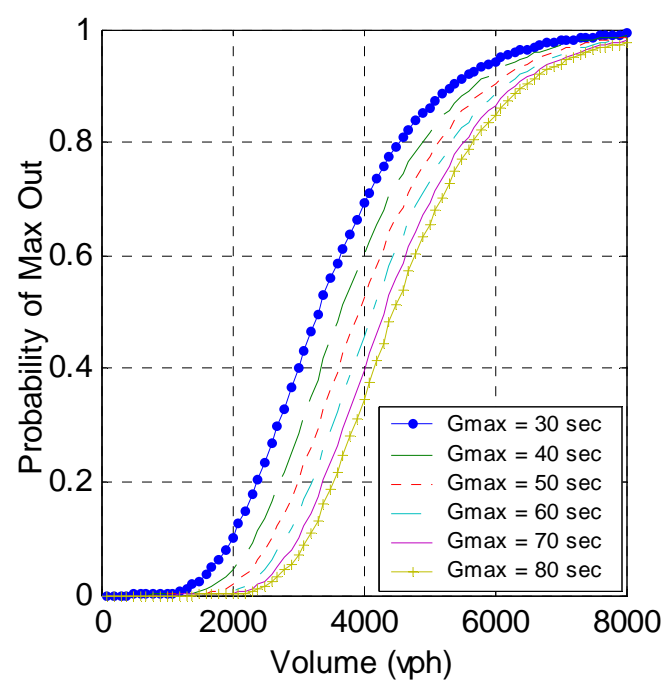

Fig. 4. Volume versus probability of max out for given maximum green time.

Figure 4 shows that increase in volume, for a fixed green max time, leads to an increase in probability of max out. For a 30 second and total traffic volume of $4600 \mathrm{vph}$, probability of max out is 0.8 . This implies that on an average 8 out of 10 cycles will max out under such volume conditions. Number of dilemma zone incursions is directly proportional to the number of max-outs. The number of vehicles facing dilemma zone increases with the increase in the number of max-outs.

A case study done by Sharma et al. (2005); indicates that the simultaneous gap out logic works well during the night when traffic volumes are low. However, during the morning, noon, and evening peaks, the percentage of max outs can be substantial, and range from $3.5 \%$ to as high as $40 \%$. High percentages of max out were usually observed during the evening peak. The $40 \% \max$ out suggests that nearly half of the cycles in that hour were forced to max out. The higher frequency of max outs during the peak periods has a negative impact on the operational efficiency during these periods as cycle length extensions may lead to excessive delays on the cross streets. The study also reported that 213 incursions occurred on the day the data was collected, with the highest hourly rate of incursions of 60 vehicles/hr. These numbers are highly significant from a safety standpoint as they indicate the number of drivers exposed to higher risk of crashes per day.

The proposed approach modifies traditional gap out logic. Under low volume conditions, all the four lanes are included in simultaneous gap out logic. But, under high volume conditions only a subset of lanes are included in simultaneous gap out logic.

In view of the objective of being responsive to the changing traffic conditions, the concept of the stochastic control methodology was formulated as follows (see Figure 5):

1. A control period of 15 minutes is used.

2. All the available data (historical and immediate past observed data) is used to predict the traffic volume in control period.

3. Optimum number of lanes to be included in the simultaneous gap out logic is chosen using following steps:

a. Volume versus probability max out plot will be used to predict the probability of max out under existing volume conditions if all four lanes are included in simultaneous gap out logic.

b. If the probability of max-out passes a certain threshold, number of lanes used in simultaneous gap out logic will be reduced .

4. The chosen control strategy would be put into operation.

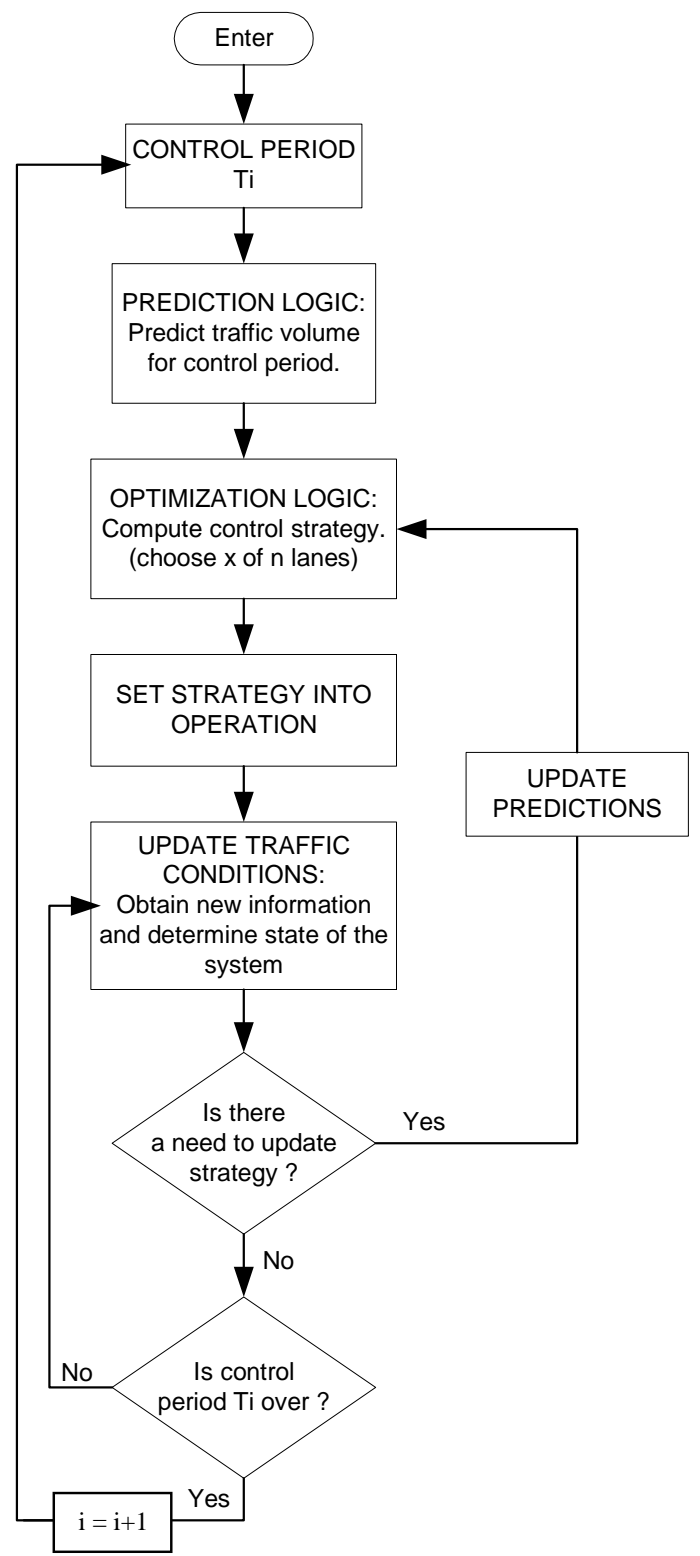

Fig. 5. Control methodology concept. 
5. While following this strategy, the system will be scanned every 5 minutes and traffic condition will be updated using the new available information.

6. Determine if a change in optimal strategy is needed; if yes, update the control strategy and repeat the process. If not, the system is continued to be scanned until the end of control time period.

\section{SIMULATION RESULTS}

VISSIM, a microscopic simulation model, was used for simulation and validation of the proposed approach. Figure 6 presents the network used for the simulation. The network consisted of a main street with two lanes in each direction with a speed limit of $55 \mathrm{mph}$ and a cross street with single lane in each direction and a speed limit of $35 \mathrm{mph}$. The controller logic for operating the signal was coded using VAP (Vehicle Actuated Programming). The volume on the main street was varied from 500 vphpln to 1400 vphpln with 300 vphpln increments. The cross street volume was kept fixed at $1000 \mathrm{vph}$. Max green time of 30 seconds and gap extension time of 4 seconds was used for the main street. All the scenarios were simulated for one hour period using three different random number seeds.

Table 1 lists the performance of the network when all-4 advance detectors are used in simultaneous gap out logic. It can be seen for low volume conditions $(500 \mathrm{vph} / \mathrm{ln}$ ) the number of max-outs are relatively low and only few vehicles are subjected to dilemma zone incursion in an hour of simulation run. But at high volumes, a higher number of vehicles are subjected to dilemma zone. In case of $1400 \mathrm{vph} / \mathrm{ln}$, 134 vehicles are subjected to dilemma zone in one hour of simulation run.

Average green of main street is a surrogate measure for the time the cross street vehicles will be delayed. During high volumes the average green approaches the max out time and the signal operates at a lower efficiency.

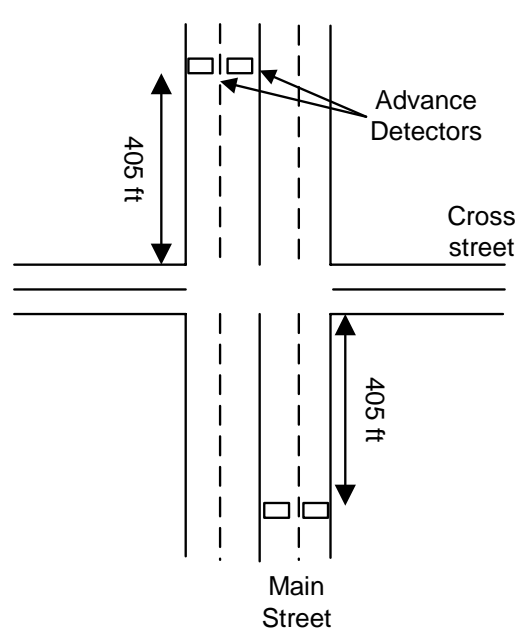

Fig. 6. Vissim simulation network.
Table 1 Simulation results when all-4 lanes included in simultaneous gap out logic

\begin{tabular}{llllllc}
$\begin{array}{c}\text { Vol } \\
\text { vph/ln }\end{array}$ & $\begin{array}{c}\text { Rand } \\
\text { No. } \\
\text { Seed }\end{array}$ & $\begin{array}{c}\# \\
\text { Cycles }\end{array}$ & $\begin{array}{c}\text { Max } \\
\text { Muts }\end{array}$ & $\begin{array}{c}\text { Prob. } \\
\text { Max } \\
\text { Outs }\end{array}$ & $\begin{array}{c}\text { \# } \\
\text { DLZ }\end{array}$ & $\begin{array}{c}\text { Avg. } \\
\text { Green } \\
\text { sec }\end{array}$ \\
\hline 500 & 42 & 92 & 8 & 0.09 & 1 & 15.91 \\
500 & 10 & 90 & 7 & 0.08 & 6 & 16.86 \\
500 & 20 & 92 & 3 & 0.03 & 3 & 15.83 \\
800 & 42 & 82 & 17 & 0.21 & 20 & 20.96 \\
800 & 10 & 80 & 27 & 0.34 & 35 & 21.84 \\
800 & 20 & 83 & 18 & 0.22 & 28 & 20.36 \\
1100 & 42 & 74 & 40 & 0.54 & 78 & 25.39 \\
1100 & 10 & 74 & 38 & 0.51 & 71 & 25.07 \\
1100 & 20 & 74 & 39 & 0.53 & 96 & 25.14 \\
1400 & 42 & 69 & 53 & 0.77 & 123 & 28.74 \\
1400 & 10 & 70 & 51 & 0.73 & 133 & 28.23 \\
1400 & 20 & 69 & 55 & 0.80 & 134 & 28.82 \\
\hline
\end{tabular}

A second set of simulation runs were performed using an advanced gap out logic. The controller logic same as the traditional gap out logic was used for the first 15 seconds of the main street green phase. During the last 15 seconds, the main street phase could gap out in case only a single vehicle was present in the dilemma zone. Table 2 lists the performance of the network using the above advanced logic, which is equivalent of using 3 of 4 lanes in last 15 seconds of the main street green phase.

Table 2 indicates that the efficiency of the signal can be improved using the advanced logic due to the reduction of average green time for main street. But, the safety of the intersection deteriorates during low volume conditions. For high volumes, both safety and efficiency of the intersection can be improved using the advanced gap out logic.

Table 2 Simulation results when all-4 lanes included in simultaneous gap out logic

\begin{tabular}{ccccccc}
$\begin{array}{c}\text { Vol } \\
\text { vph/ln }\end{array}$ & $\begin{array}{c}\text { Rand } \\
\text { No. } \\
\text { Seed }\end{array}$ & $\begin{array}{c}\# \\
\text { Cycles }\end{array}$ & $\begin{array}{c}\# \\
\text { Max } \\
\text { Outs }\end{array}$ & $\begin{array}{c}\text { Prob. } \\
\text { Max } \\
\text { Outs }\end{array}$ & $\begin{array}{c}\# \\
\text { DLZ }\end{array}$ & $\begin{array}{c}\text { Avg. } \\
\text { Green } \\
\text { sec }\end{array}$ \\
\hline 500 & 42 & 97 & 0 & 0.00 & 27 & 13.83 \\
500 & 10 & 97 & 0 & 0.00 & 26 & 13.88 \\
500 & 20 & 98 & 0 & 0.00 & 33 & 13.67 \\
800 & 42 & 95 & 0 & 0.00 & 42 & 14.83 \\
800 & 10 & 94 & 0 & 0.00 & 51 & 15.32 \\
800 & 20 & 94 & 0 & 0.00 & 49 & 15.29 \\
1100 & 42 & 87 & 2 & 0.02 & 69 & 18.16 \\
1100 & 10 & 89 & 0 & 0.00 & 64 & 17.29 \\
1100 & 20 & 89 & 1 & 0.01 & 67 & 17.34 \\
1400 & 42 & 82 & 7 & 0.09 & 84 & 20.65 \\
1400 & 10 & 84 & 6 & 0.07 & 88 & 19.88 \\
1400 & 20 & 84 & 3 & 0.04 & 76 & 19.79 \\
\hline
\end{tabular}




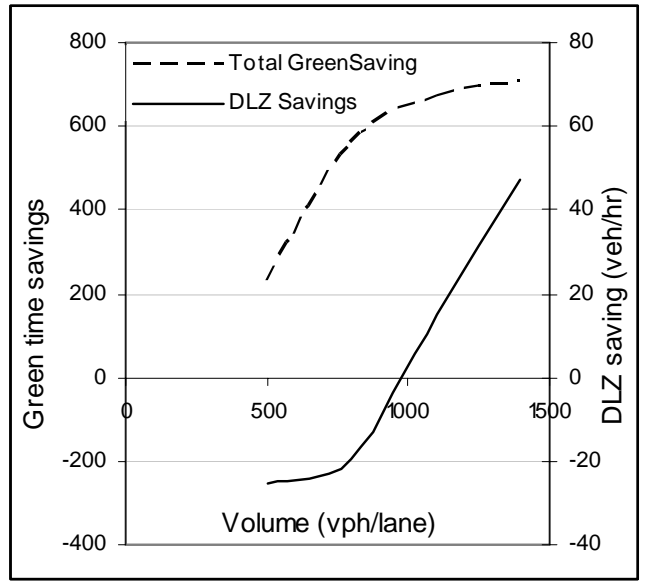

Fig. 6. Green savings and reduction in dilemma zone incursions by switching from traditional gap out logic to the advanced gap out logic.

Figure 6 shows the improvements in safety and efficiency when the advanced gap out logic is used. It can be seen when the volume per lane is below $1000 \mathrm{vph} / \mathrm{ln}$ the advance gap out logic have better efficiency but poor safety characteristics as compared to the traditional logic. But if the traffic volume is more than $1000 \mathrm{vph} / \mathrm{ln}$ advanced gap out logic will have superior safety and efficiency.

Based on the above results the threshold to switch from all-4 lanes to 3 of 4 lanes logic can be set at $1000 \mathrm{vph} / \mathrm{ln}$. When overall volume of $4000 \mathrm{vph}$ is detected on all the 4 lanes, the control strategy using three of four lanes will be implemented.

\section{CONCLUDING COMMENTS}

The results obtained from microscopic simulation validates that both safety and efficiency of operation at a high speed signalized intersection can be improved by using traffic adaptive advanced gap out logic (described in Figure 5). The above logic should also be tested on the field data before wide scale implementations.

The success of the traffic adaptive approach will depend upon the placement of the advance detectors and variability in volumes at a specific site. Traffic engineer needs to carefully study the amplitude and frequency of variation of traffic volumes before implementation of the proposed logic. Both historical and present data should be analysed to improve the performance of the traffic adaptive approach. If correctly implemented, the proposed approach can provide a significant improvement in safety and efficiency of operation at high speed isolated intersections.

\section{REFERENCES}

Bonneson, J., D. Middleton, K. Zimmerman, H. Charara, and M. Abbas (2002). Intelligent Detection-Control System for Rural Signalized Intersections. Report No. FHWA/TX-03/4022-2. Texas Department of Transportation, Austin, Texas.

Casella, G. and R Berger (2002). Statistical Inference. Second Edition. Duxbury Press, Pacific Grove, CA.

Crawford, A (1962). Driver Judgment and Error during the Amber Period at Traffic Lights. Ergonomics, Vol. 5, 513-532.

Gazis, D., R. Herman, and A. Maradudin (1960). The Problem of the Amber Signal Light in Traffic Flow. Operations Research, Vol. 8, No. 1,112132.

Herman, R (1963). Problem of the Amber Signal Light. Traffic Engineering and Control, Vol. 5, 298-304.

ITE (1999). The Traffic Safety Toolbox: A Primer on Traffic Safety. Institute of Transportation Engineers, Washington, DC.

McCoy, P. and G. Pesti (2002), Dilemma Zone Protection with Advance Detection and Active Warning Signs. Annual meeting of the Institute of Transportation Engineers.

Middleton, D., D. Jasek, H. Charara, and D. Moris (1997). Evaluation of Innovative Methods to Reduce Stops to Trucks at Isolated Signalized Intersections. Report No. TX-97/2972-S. Texas Department of Transportation, Austin, Texas.

Olson, P. and R. Rothery (1972). Deceleration Levels and Clearance Times Associated with the Amber Phase of Traffic Signals. Traffic Engineering, Vol. 42, No. 4, 16-19.

Retting, R., R. Ulmer, and A. Williams (1999). Prevalence and Characteristics of Red-LightRunning Crashes in the United States. Accident Analysis and Prevention. Vol. 31, 687-694.

Saito, T., N. Ooyama, and K. Sigeta (1990). Dilemma and option zones, the problem and countermeasures-characteristics of zones, and a new strategy of signal control for minimizing zones. Road Traffic Control, Third International Conference.

Sharma, A., D. Bullock, and S. Peeta (2005). Limitations of Simultaneous Gap Out Logic, Transportation Research Record, TRB, National Research Council, Washington, DC, TRB Paper ID\# 06-1309.

Sheffi, Y. and H. Mahmassani (1981). Model of Driver Behavior at High-Speed Signalized Intersections. Transportation Science, Vol. 15, No. 1, 50-61.

Zegeer, C (1977). Effectiveness of Green-Extension Systems at High-Speed Intersections. Research Report 472, Kentucky Department of Transportation.

Zegeer, C. and R. Deen (1978). Green-Extension Systems at High-Speed Intersections. ITE Journal, Nov. issue, Washington, DC. 